Publicación semestral. ISSN 2215-4906

Volumen 81 - Número 1

Julio - Diciembre 2021

\title{
Entrelazando caminos. Mis experiencias de colaboración en la investigación artística al hacer comunidad
}

\author{
Intertwining Paths. My Experiences of Collaboration \\ in Artistic Research in Community Making
}

Angélica Jarumi Dávila López

DOI 10.15517/es.v81i1.47648

(c) (i) (2) Esta obra está bajo una licencia Creative Commons
By NG ND Reconocimiento-No comercial-Sin Obra Derivada 


\title{
Entrelazando caminos. Mis experiencias de colaboración en la investigación artística al hacer comunidad
}

\author{
Intertwining Paths. My Experiences of Collaboration \\ in Artistic Research in Community Making
}

\author{
Angélica Jarumi Dávila López ${ }^{2}$ \\ Universidad Nacional Autónoma de México \\ Ciudad de México, México
}

Recibido: 12 de febrero de 2021 Aprobado:16 de marzo de 2021

\begin{abstract}
Resumen:
El presente artículo aborda la propuesta de la investigación artística como eje de la asignatura de Investigación en Artes Visuales que la autora imparte en la Maestría en Artes Visuales del Posgrado en Artes y Diseño de la Universidad Nacional Autónoma de México. A partir de la labor realizada con los estudiantes, se revalora la importancia del proceso creativo y se enfatiza la idea de la experiencia como elemento colaborativo para hacer comunidad durante el desarrollo del proyecto de investigación. Para ello, se retoman fuentes tanto teóricas como experienciales, haciendo referencia a investigadores especialistas en el tema y a las indagaciones derivadas de experiencias vividas en diversos momentos de la formación y el desempeño profesional de la autora, por lo que se trata de un proceso deconstructivo de lo que se solía entender y desarrollar como investigación. Además, el artículo se complementa con tres imágenes a manera de registro de bitácora.
\end{abstract}

Palabras clave: investigación; proceso de aprendizaje; artes visuales; comunidad; experiencia

1 Este trabajo se realizó con el apoyo del Programa UNAM-DGAPA-PAPIME PE404520.

2 Docente e investigadora en la Facultad de Arte y Diseño de la Universidad Nacional Autónoma de México. Maestra en Artes Visuales por la Universidad Nacional Autónoma de México. ORCID: 0000-0001-7076-3466. Correo electrónico: jdavila@fad.unam.mx 
Entrelazando caminos. Mis experiencias de colaboración

Dossier

en la investigación artística al hacer comunidad

\begin{abstract}
:
This article addresses the proposal of Artistic Research as the axis of the Research in Visual Arts subject that the author teaches in the Master of Visual Arts of the Postgraduate Degree in Arts and Design at Universidad Nacional Autónoma de México (UNAM), based on the work carried out with the students, the importance of the creative process is revalued and the idea of experience as a collaborative element to form a community is emphasized during the development of the research project. For which, both theoretical and experiential sources are taken up, referring to researchers specializing in the subject, and to the inquiries derived from experiences lived at various moments of the author's training and professional performance so it is a process deconstructive of what used to be understood and developed as research. Complemented with three images as a logbook.
\end{abstract}

Keywords: research; learning process; visual arts; community; experience 


\section{De inicio}

Investigación ... pensar las distintas formas en que el mundo puede ser entendido

(Smith³, 2016, p. 33).

Antes de comenzar es importante aclarar que, si bien los acontecimientos en la vida se desarrollan de forma secuencial (cronológicamente), al momento de escribir este texto, el cual para mí es un ejercicio de reflexividad sobre mi proceso creativo al desarrollar una investigación, los sucesos no necesariamente mantienen el esquema positivista de la linealidad, ya que las ideas entrecruzan momentos de reflexión que vienen y van entre los hechos que conforman las experiencias. Durante el texto se mencionan, en varias ocasiones, las actividades relacionadas con mis estudios de maestría y las realizadas en mi práctica profesional como docente. Es un ir y venir entre ambos procesos de acción, pues están entrelazados tanto en la práctica como en la reflexión y son estos los que dan cuerpo al presente artículo.

En el ámbito académico, acercarse a conceptos cuyas definiciones se encuentran aún en desarrollo brinda la gran posibilidad de experimentar y adentrarse en terrenos poco conocidos. De ese modo, en el camino uno va encontrándose con quienes también andan siguiendo el rastro de una investigación que pareciera invitar al ánimo deconstructivo de las formas y de los contenidos, lo que a su vez conlleva a las intencionalidades de quienes pretendan servirse de ella al mismo tiempo que la constituyen. Es entonces que me permito compartir sobre lo que he encontrado al transitar por este camino.

3 Linda Tuhiwai Smith (Ngati Awa y Ngati Porou, maorí) es una estudiosa de la educación y crítica del colonialismo persistente en la enseñanza e investigación académicas. Uno de los esfuerzos más importantes de Smith es vincular la historia de la conquista y colonización europeas con el desarrollo del pensamiento científico, basado en la deshumanización y apropiación de los pueblos indígenas de todo el mundo. Ha demostrado que, aunque no siguen el 'método científico', las epistemologías indígenas no son menos rigurosas y dignas de respeto. Como consecuencia, ha pedido que la academia reconozca que los conocimientos indígenas no deben subordinarse a los conocimientos académicos, sino que deben ser respetados como formas paralelas de conocimiento (Battell Lowman \& Barker, s.f.). Retomar a esta autora es consecuente con las reflexiones que he generado a partir de mi proyecto de investigación de la maestría, las cuales han sido esenciales en mi desempeño como docente, sobre ello hablará más adelante en este texto.

ESCENA. Revista de las artes, 2021, Vol. 81, Núm. 1 (julio-diciembre), pp. 304-319 
Mi experiencia de aprendizaje al cursar la maestría me permitió re-conocerme en el ámbito tanto profesional como en el personal, ya que me brindó la oportunidad de desarrollar un trabajo investigativo cuyo proceso me develó la existencia de la investigación artística, sin lograr entender de entrada lo que es esta en realidad. Han pasado algunos años desde esa develación; el proyecto tuvo una conclusión "formal", obtuve el grado y mantuve relación estrecha con los "resultados". Asimismo, entre los senderos que han abierto el paso a múltiples posibilidades de relación, la de profundizar en uno de ellos está latente desde hace un par de años. Mismos años en los que he tenido la oportunidad de compartir saberes y sentires como integrante del grupo de investigación Mitote Intervenciones Críticas Desde el Arte Contemporáneo ICDAC, ${ }^{4}$ en el cual hemos establecido la investigación artística como una de nuestras líneas de indagación.

Esta modalidad de investigación es un concepto relativamente nuevo, pero del que se cuenta ya con ciertos antecedentes - quizás el más directo es el trabajo de Henk Borgdorff desarrollado en su artículo El debate sobre la investigación en las artes (2019)5, de donde podríamos considerar que parte la línea que aquí se plantea-. Por lo tanto, la definición de investigación artística aún se encuentra en construcción. Sin embargo, esta necesidad por definir ha dado pauta a reflexionar sobre ciertas estructuras dentro del ámbito artístico, en las cuales se requiere precisar con el fin de validar. Esto recuerda los postulados derivados del cientificismo y del positivismo, ya consagrados por la llustración ${ }^{6} \mathrm{y}$, por ende, propios de la modernidad.

4 Para mayor información sobre el grupo puede consultarse la página https://mitote.org/

${ }^{5}$ El antecedente de esta propuesta de investigación lo encontramos claramente en el trabajo de Henk Borgdorff del Amsterdam School of the Arts, con su conocido artículo "El debate sobre la investigación en las artes" (2019), en el cual plantea con gran claridad las diferencias entre: a) investigación sobre las artes, b) investigación para las artes y c) investigación en las artes. Es con el último inciso con el que la investigación artística tendría un acercamiento significativo o, quizá es mejor decir, un referente, ya que en él expresa el concepto de "perspectiva de la acción" o "perspectiva inmanente", es decir, se trata de una investigación que no separa al sujeto del objeto, a la vez que no contempla distancia entre el investigador y la práctica artística, así como tampoco existe separación entre teoría y práctica (Borgdorff, 2019). Como se verá más adelante en este texto, estos elementos estarán presentes en la propuesta de la investigación artística.

6 Recordemos el postulado del pensamiento ilustrado del siglo XVIII "orden, razón y progreso", momento en que la oficialidad se hace presente a partir de la consagración del diccionario y la enciclopedia, herramientas cuya función esencial fue y es ordenar y clasificar el conocimiento. 
Volviendo sobre el tema, existen lazos entre las posibles fundamentaciones de la investigación artística y algunas de las propuestas del arte contemporáneo. De manera que, actualmente, se está teorizando sobre este concepto, a la vez que él mismo se está autoconstituyendo a través de la práctica artística contemporánea. No obstante, ello no es razón para no encontrar sus bases conceptuales en periodos anteriores, en los cuales probablemente no se tenía la intención, o en ocasiones la claridad, de que se estaba realizando este tipo de investigación, pero que dan cuenta de referencias a las características de la misma.

Por lo anterior, resulta importante rastrear el proceso mismo de su gestación, así como tratar de indagar acerca de lo que le es propio. Hoy se cuenta con una base de ello por parte de estudiosos europeos. De manera que, ahora compete realizar el ejercicio de recopilar y estructurar el trabajo realizado de este lado, ya que, en gran parte del ámbito propiamente académico de las artes, aún es muy poco conocido el hecho mismo de que exista esta propuesta de investigación. Es tarea ardua lograr clarificar en lo posible su constitución y visibilizar los alcances ya obtenidos, con el fin de nutrir y ampliar las posibilidades del quehacer investigativo en las artes.

Como parte de mi proceso de investigación en este respecto, he trabajado con material ya recopilado, organizado, analizado e inclusive comprobado ${ }^{7}$ - preferiría $^{2}$ no usar ese término, sino, más bien, llevado a la práctica ${ }^{8}-$. A partir de lo anterior, ha quedado en evidencia la relevancia de compartir, de realizar intercambio de ideas y de lograr retroalimentación en grupo. Como se verá más adelante en este texto, el ejercicio de reflexividad (Calderón \& Hernández, 2019) es un elemento crucial en la investigación artística y solo es posible llegar a ella a través del intercambio con otro(s). Es por ello que, para la realización de esta investigación, ha resultado fundamental la reciprocidad de voces que otorga el trabajo en colectivo al fomentar la reflexión en comunidad.

Ramón Xirau (2003) lo desarrolla en el apartado “V. El camino de la crítica: La llustración”, incluido en su libro Introducción a la historia de la filosofía, editado por la Universidad Nacional Autónoma de México.

7 Este es un término más propio de la investigación de índole cientificista y, si bien no lo considero el más adecuado, lo dejo, ya que quizá resulta favorable el "comparativo" para comprender que hay ciertas similitudes en los procesos de investigación.

8 Esto lo retomaré más adelante, pues tiene que ver totalmente con los resultados derivados de impartir la asignatura de Investigación en Artes en el posgrado de Artes y Diseño de la FAD, UNAM.

ESCENA. Revista de las artes, 2021, Vol. 81, Núm. 1 (julio-diciembre), pp. 304-319 
Como ya comenté, desde hace alrededor de cinco años, soy parte del grupo de investigación Mitote ICDAC. La solidez de este trabajo colaborativo es ya referencia en el campo de estudio de la investigación artística, razón por la cual se le toma en cuenta para participar en eventos relacionados con el tema y como referencia en publicaciones sobre el mismo. Es necesario destacar que como grupo se ha logrado estrechar lazos de colaboración con instituciones, artistas y otros grupos de investigación, tanto nacionales como extranjeros, interesados e inmersos en el tema, situación que beneficia y genera transversalidades en el proceso de la indagación en desarrollo.

Tal es el caso del trabajo realizado con la Universidad Veracruzana, a través del Seminario Permanente de Investigación Artística SPIA ${ }^{9}$, con el que se han celebrado dos Encuentros sobre Investigación Artística. Asimismo, se han llevado a cabo proyectos con el Museo Universitario de Arte Contemporáneo (MUAC) de la Universidad Nacional Autónoma de México (UNAM), como las Jornadas de Desintoxicación, donde ha tenido lugar el diálogo compartido entre docentes de diversas instituciones, con el fin de compartir experiencias acerca de los procesos de enseñanza e investigación en el medio de las artes. De igual forma, con el grupo "Inventamos o Erramos" de la Universidad Autónoma de la Ciudad de México $(\mathrm{UACM})^{10}$ se han generado estrechos lazos colaborativos con respecto a los procesos de educación en Latinoamérica. Algo similar ha sucedido con el Movimiento Mexicano para la Escuela Moderna (MMEM) ${ }^{11}$, con quienes se han presentado encuentros sobre educación artística también.

Con todos ellos se ha colaborado en diversas formas y como resultado de dichas colaboraciones se han ido entrelazando las ideas y las experiencias que dan cuerpo a las reflexiones en conjunto, pero también de manera personal. Estas son las mismas experiencias que tejen las líneas de investigación del grupo y sus integrantes, cual hilos que si bien dan cuenta en lo individual de un color propio, dotan de volumen y textura al fortalecido tejido que se enuncia en la búsqueda de abonar, compartiendo con el colectivo, temas impe-

9 Para conocer más sobre este Seminario Permanente de Investigación se puede consultar su sitio https://investigacionspia.wordpress.com/

10 Para conocer más sobre este grupo de investigación se puede visitar el siguiente sitio https:// oinventamosoerramosblog.wordpress.com/

${ }^{11}$ Para conocer más sobre el trabajo realizado, se puede visitar el sitio http://www.mmem.org.mx/ 
rativos en el devenir de la educación y la investigación artísticas. Para conocer un poco sobre lo compartido y generado se pueden visitar los sitios en internet referidos a pie de página de este documento.

En otro orden de cosas, un factor esencial en este proceso ha sido y es la interacción con estudiantes, ya sea dirigiendo investigaciones de tesis o al ser asesor en investigaciones de producción artística, ser tutor de grupo o de forma individual, pero, sobre todo, al impartir clase. Resulta crucial el intercambio directo con los estudiantes del Posgrado en Artes y Diseño de la UNAM, en la orientación de investigación en Artes Visuales. Esto debido a que el programa de dicha materia lo conformé con la intención de abordar la investigación artística desde sus bases y al cual titulé Investigación desde la experiencia, revaloración del proceso creativo.

Ha sido en esta actividad en la que se ha logrado con mayor énfasis la relación con el tema, ya que, como el nombre de la asignatura lo indica, se enfoca por completo al área de investigación. Además, la oportunidad de compartir con un grupo cuyo número de asistentes es reducido a no más de 8 estudiantes, brinda la posibilidad del trabajo específico con cada uno y su proyecto, lo que a su vez también propicia el intercambio de ideas y reflexiones con el fin de retroalimentar en colectivo.

\section{Comunidad: Conjunto de personas que poseen características o intereses comunes}

La comunidad es un concepto que me interesa comentar, debido a que es una estructura que demanda el trabajo colaborativo por el bien común. Esto genera un ambiente de confianza en el que cada quien tiene el espacio para hablar y ser escuchado, intercambiar puntos de vista y propiciar una crítica constructiva que abone con fundamento a los proyectos de los compañeros. Para el enfoque planteado en clase, esto es de extrema importancia ya que, si bien estamos en un contexto académico institucional, estamos también en un espacio que corresponde, en ese mismo contexto, al área de las humanidades, más específico aún al de las artes, teniendo en claro que en este se aborda a la estética y por ende a lo sensible. Por esta razón, la confianza es esencial para compartir ideas, pero también sentires, ${ }^{12}$ debido a que es el conjunto de ambos lo que ha dado pie al proyecto de investigación.

${ }^{12}$ Desde el Mitote ICDAC hemos trabajado en aplicar y desarrollar el concepto sentipensante, propuesto por el sociólogo Orlando Fals Borda y ampliamente difundido por el escritor Eduardo 
Es la investigación artística un tipo de investigación que tiene muy en cuenta la relevancia de dicho conjunto, pues de entrada no plantea un único método para llevarla a cabo, sino que, por el contrario, plantea que hay múltiples métodos, tantos como se necesiten, dado que dependiendo de los objetivos y las intencionalidades se adaptarán las estrategias necesarias. Esto podría hacer pensar, entonces, que cada artistainvestigador simplemente hace el intento y logra el cometido. Resultaría increíble, de ser así, sin embargo, con lo que nos hemos encontrado es que, a partir de toda la estructura académica resultado de un proceso de introyección que da por establecida nuestra formación escolar desde el kínder hasta la universidad, no es tan fácil. Y no lo es porque en esa línea, por lo general, se tiende a realizar una investigación de índole cientificista propia del nivel de posgrado en la tradición académica.

Es entonces que en la investigación artística se manifiesta un ánimo deconstructivo, ${ }^{13}$ dado que de entrada plantea romper con lo preestablecido, desdibujar los límites tan cuadrados que suelen ser sumamente protocolarios y poco flexibles a las complejidades ${ }^{14}$ que hoy día dan cuerpo a la investigación en el área de las artes. Se propone buscar otras formas de llevarla a cabo. No es fácil, por lo que es ahí en donde el trabajo docente en el posgrado ha resultado el sitio ideal para estudiar, experienciar y desarrollar, a la vez que, en conjunto, aclarar cuales son los elementos, las características y los fines de este tipo de investigación. El cómo realizar dichas acciones me lleva a retomar algunas de las reflexiones generadas en el encuentro de investigación artística Reflexiones Indisciplinadas Segunda

Galeano, en el que se refiere sobre el pensar con la cabeza y el corazón como factor de convivencia grata y fructífera con las/os vecinas/os y la naturaleza (Vivas, 2020). Aplicado a nuestro contexto investigativo, resulta totalmente acorde a las intencionalidades que conforman los proyectos de la investigación artística.

${ }^{13}$ El concepto de deconstruir de acuerdo con la Real Academia Española (2021) significa: "deshacer analíticamente los elementos que constituyen una estructura conceptual”. Además, es un término acuñado por el filósofo postestructuralista Jacques Derrida, el cual se entiende como un intento de reorganizar de cierto modo el pensamiento occidental.

${ }^{14}$ Al referir al concepto de complejidad, es necesario señalar que se toma como referencia el planteamiento de pensamiento complejo que desarrolló Edgar Morin (Ruíz \& Torres (2016), donde es un hecho que la investigación en artes visuales se nutre de otras áreas del conocimiento de una forma holística.

ESCENA. Revista de las artes, 2021, Vol. 81, Núm. 1 (julio-diciembre), pp. 304-319 
vuelta para la acción, ${ }^{15}$ en el que participamos el Seminario Permanente de Investigación Artística (SPIA) y el Mitote ICDAC, donde se resaltó la idea sobre el proceso pedagógico que implica la investigación. Allí se planteó que la experiencia pedagógica, la cual desborda los límites de lo educativo, al buscar eliminar la jerarquía de maestro-estudiante para generar un acompañamiento conjunto en el que el diálogo es la base, con el fin de fomentar la reflexividad y tomar como herramienta esencial el registro del proceso creativo a la par de un ejercicio de recapitulación, llevará a poder enunciar los procesos y cuestionar, a su vez, los propios límites. Para ello, deberá de asumirse un compromiso con el proyecto que se realice, pensando en las implicaciones que su ejecución tendrá en su entorno inmediato.

La idea sería entonces lograr una integración de la teoría y la práctica. La primera será en esencia reflexiva con el fin de lograr la conexión que dotará de singularidad a la producción artística. Es evidente que se busca romper, des-estructurar los procesos ya aprendidos, diluir las dicotomías y consolidar a la investigación artística como un puente, una especie de zona de contacto donde se relacionan los campos de conocimiento, para plantear una intersección donde se desarrolle, en palabras de la Dra. Natalia Calderón, especialista en el tema, una investigación de carácter accesible, transparente y compartible, que se cuestione a sí misma y que, por lo tanto, se redefine constantemente.

\section{La experiencia como detonante}

Es necesario un breve paréntesis antes de continuar con las indagaciones acerca de las características de la investigación artística. Haré referencia a mi propio proceso creativo durante mis estudios de maestría en Artes Visuales, ya que fue a partir de reflexionar sobre ello que he logrado desarrollar en parte mi labor docente en el posgrado.

La investigación realizada cambió varias veces de título y se transformó en varias ocasiones, pero fue finalmente una experiencia de vida la que constituyó el detonante crucial para vislumbrar hacia dónde quería llevar el proyecto y desde qué enfoque realizarlo. Cuando al fin logré esclarecer mis intenciones, comenzó el fluir, en el último año en que trabajé en ello para dar cuerpo y voz a mi propuesta.

${ }^{15}$ Encuentro de Investigación Artística SPIA-ICDAC Reflexiones Indisciplinadas, segunda vuelta para la acción, el 9 de septiembre de 2019, en la Sala de Videoconferencias de la FAD-Xochimilco, UNAM, en México.

ESCENA. Revista de las artes, 2021, Vol. 81, Núm. 1 (julio-diciembre), pp. 304-319 
Así desarrollé la tesis para obtener el grado de Maestría en Artes Visuales titulada Sobre cómo la mirada mágica pone en escena al ser, a la vez que este se devela en el proceso creativo (Dávila, 2015). El planteamiento, ya latente en el título, es la revaloración del proceso creativo y la importancia de la experiencia en el proceso de investigación-producción artística. Realicé una reflexión sobre ello a partir de la práctica de lo fotográfico y del dibujo, cuyo tema fue la simbología del mito del venado azul de los Wixaritari, comúnmente conocidos como huicholes. ${ }^{16}$ Para lo cual, además de realizar investigación de tipo bibliográfica, me dirigí directo a las fuentes, es decir, a la convivencia con una comunidad Wixárika en la sierra madre occidental de México, más específicamente en la Sierra del Nayar en el estado de Tepic, Nayarit, hacia el noroeste del territorio nacional.

Digo convivir porque, a pesar de que la primera intención - aunque algo fugaz- fue académica, se convirtió en parte de mi vida por el grado de confianza y cariño que nuestro lazo de conexión ha alcanzado. Para algunas personas en el ámbito profesional, yo realicé etnografía participativa; sin embargo, lo cierto es que nunca me acerqué a dicha rama de estudio, sino que incluso descubrí el término en la ocasión en que me trataron de enunciar desde él. Lo que sé es que las experiencias compartidas en esa pequeña comunidad de la sierra han tocado de manera profunda en mi ser, en mis pensares, mis sentires e inclusive algunos de mis saberes han transmutado de tal forma que hoy, después de un ejercicio de recapitulación y de reflexión, puedo escribir estas líneas.

No pretendo dar un resumen de lo que versó dicha tesis, enunciarla es ya un referente importante para lo que este texto tiene como objetivo. Por tanto, encausando de nuevo las letras hacia el cometido, solo debo acotar que las experiencias de intercambio con las personas de la comunidad -las mujeres, los niños, las abuelas, los hombres-, las conversaciones, las risas, los silencios, las miradas, el compartir sus espacios, su territorio, su lengua, su ser y su sentir me brindaron la oportunidad de conocer, de saberme en ellos y ellas, de comprender mi relación con el mundo de otra manera, una distinta al tiempoespacio que me había tocado vivir hasta entonces.

${ }^{16}$ La cultura Wixárika tiene sus asentamientos en lo alto de la Sierra Madre Occidental, en los lindes de los estados de Jalisco, Nayarit, Zacatecas y Durango, México. Es un pueblo con una tradición milenaria, cuya principal característica dentro de los grupos indígenas de México es el intentar mantener sus usos y costumbres lo más vivas posible.

ESCENA. Revista de las artes, 2021, Vol. 81, Núm. 1 (julio-diciembre), pp. 304-319 
Para esa época yo llevaba más de 10 años desarrollando la labor docente. Si bien desde siempre he creído en la estrecha relación de la propuesta artística y lo sensible, en que la experiencia es su base, estudiar la maestría y desarrollar el proyecto ya mencionado me dieron la posibilidad de ampliar mis reflexiones desde el ámbito profesional y personal, de lo que vislumbro a la distancia, de lo que he aplicado en las clases en el posgrado desde hace poco más de un año y medio que me invitaron a impartir clase en él. Es ahora que hago a conciencia el ejercicio de reflexividad, cuando puedo inferir lo siguiente como resultado de mi compartir:

- El acercamiento es muy valioso; desdibujar los "límites" que marcan las jerarquías de poder "del que sabe más", con el fin de evidenciar que quienes dialogan tienen algo que aportar, que compartir. Se aprende de todo y de todos.

- Saber escuchar se deriva por completo de lo anterior.

- Construir un ambiente de confianza donde se valora lo enunciado, sin juzgar, con la idea de compartir, de dialogar, a la vez que disentir, respetar las ideas del otro, para nutrirse y así poder retroalimentar.

- La constancia es esencial. Para que el intercambio sea fluido, lo mejor es que sea constante y esto, a su vez, consolide el ambiente de confianza.

- Dar espacio a lo que se piensa es importante, pero también lo es dar cabida a lo que se siente. La parte sensible y emotiva tiene un lugar primordial en el desarrollo de la propuesta artística y con el paso del tiempo y las transformaciones económicas, políticas y sociales, las mayoría de las veces se va quedando al margen e incluso se llega a excluir. Se presenta como cliché y se demerita lo que en realidad es parte de la raíz. Lo que se conoce tiene un carácter muchas veces académico, por lo que es tiempo de dar su lugar a que lo que se siente, ya que también nutre la retroalimentación. Las experiencias tienen ahí gran parte de su esencia. (Esto tiene una relación directa con el concepto sentipensante).

Todo lo anterior ya era en cierta forma elemento de mis actividades, pero la potencialidad de hacerlo más consciente y aplicarlo con intenciones definidas y encausadas en las sesiones de clase del posgrado me ha permitido compartir mi experiencia para nutrir la de los estudiantes. Aunque resulte algo reiterativo, me es primordial enfatizar que lo enlistado arriba es resultado de mi experiencia al convivir con una comunidad y lo he llevado al espacio académico con el fin de construir una con las y los estudiantes. 
Es importante señalar que el registro de mi proceso creativo fue esencial para que la lista anterior lograse ser conformada. Al contar con la posibilidad de recapitular sobre lo vivido, el ejercicio de reflexividad dio paso a la concreción de los puntos aquí presentados. La herramienta maestra de esto fue la bitácora, compañera inseparable de mi transitar por el territorio investigado y de la que hablaré más adelante con mayor detalle.

Imagen 1. Comunidad

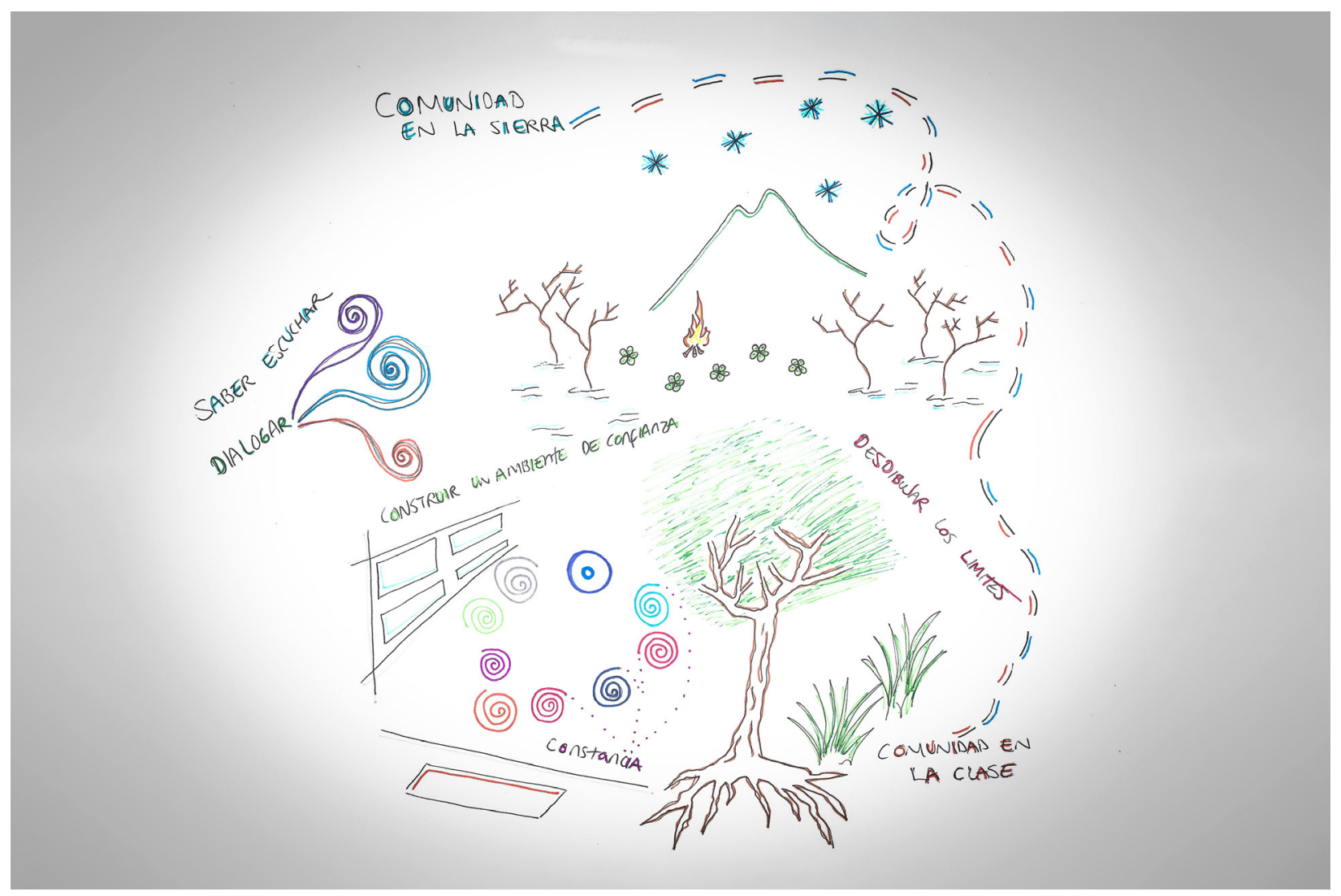

Fuente: Elaboración propia.

Por lo pronto, siendo consecuente con tan maravilloso medio de registro, se presenta la reflexión visual (a manera de bitácora) en la imagen 1, donde se muestra la referencia al espacio de la comunidad en la Sierra del Nayar y el espacio de la comunidad en la clase. Este último no se cerraba al salón asignado, pues también realizamos sesiones en espacio abierto dentro de las mismas instalaciones de la UNAM. 
Entrelazando caminos. Mis experiencias de colaboración

Dossier

en la investigación artística al hacer comunidad

\section{Desde la experiencia: hacer comunidad durante el proceso}

Al ser la maestra, tengo un trecho del camino ya andado, es decir, mi quehacer como artista-investigador ha profundizado un poco más en ello. Si a eso le sumamos la experiencia recopilada durante 15 años como docente en el área de la estética, la historia y la teoría del arte, se entiende el que me corresponda guiar el proceso.

Aquí es importante aclarar que los estudiantes entran al posgrado con la idea de realizar investigación. A veces ya traen la idea y el conocimiento acerca de la existencia de la investigación artística como concepto y otras veces no. Sin embargo, por la naturaleza de sus intencionalidades, intuyen que no es a través de la metodología de base cientificista tradicional que lograran los resultados deseados. Es entonces cuando les digo de qué va el curso: pongo sobre la mesa la propuesta de realizar el proyecto de investigación bajo la guía de los postulados de la investigación artística; les explico a grandes rasgos la información que ya se tiene teorizada al respecto y les comento que, a la vez, iremos investigando y desarrollando a qué refiere como concepto. Es muy interesante que, hasta el momento, la respuesta ha sido un sí tajante, pues, como ya decía, sus búsquedas tienen necesidades donde la parte sensible reclama tener una participación consciente.

Por ello, gran parte de mi labor ha sido y es brindar un acompañamiento que se nutre de los procesos de todos los compañeros, mi bagaje de sapiencia y experiencia me brinda la oportunidad de ayudarles a encontrar o clarificar sus propias estrategias de investigación, mismas que se ven nutridas y retroalimentadas a través del intercambio con los otros a partir de las dinámicas generadas en las sesiones de clase para lo cual, me he servido un tanto de los alcances que hemos logrado en el Mitote ICDAC sobre la educación artística, así como de la información obtenida de diversas fuentes. Lo importante aquí es señalar que las estrategias pedagógicas en el aula ${ }^{17}$ juegan un papel esencial para lograr el ambiente de confianza del que hablaba en un inicio, así como para ser consecuente con los propios elementos y características de la investigación artística. Sobre estas últimas hemos comentado y reflexionado en clase, a partir de lo que hasta ahora hemos ubicado lo siguiente:

${ }^{17}$ Para conocer algunas de ellas y cómo funcionan, se puede consultar la página del grupo referida anteriormente.

ESCENA. Revista de las artes, 2021, Vol. 81, Núm. 1 (julio-diciembre), pp. 304-319 
- Múltiples Metodologías (no unívoca).

- Fuentes de información que abarcan amplitud de posibilidades. Es conveniente poner de manifiesto la relación estrecha con el planteamiento del pensamiento complejo que propone Edgar Morin, ya que en él expone la necesidad y la oportunidad al mismo tiempo de abarcar un tema desde diferentes ángulos, lo que a su vez incluye diversas áreas del conocimiento, dando paso a la interdisciplina (Ruíz \& Torres, 2016).

- Reflexión sobre la función del arte.

- Experimentación.

- Procesos investigativos (sentido crítico de la historia). La referencia directa a ello es la propuesta de Santiago Vera Cañizares docente de la Universidad de Granada, España, quien en su libro Proyecto artístico y territorio (Vera, 2004) propone a la investigación como un viaje. Además plantea la posibilidad de desarrollar la investigación no solo desde la vertiente lineal y cientificista, característica de la modernidad, sino que también desde lo que denomina como el proyecto antropológico existencial, donde se da cabida e importancia a ubicar en dónde fue que se detonó el tema de nuestra investigación, a partir de una experiencia en nuestro cotidiano, para posteriormente sacarlo de ahí, re-significarlo y decidir el enfoque desde el que se ha decidido "recorrerlo", para seguir con la metáfora del viaje que él mismo plantea. Esto se conecta de forma muy natural con lo que se plantea un poco más atrás en este texto sobre la pertinencia del pensamiento complejo propuesto por Edgar Morin.

- Escritura como proceso necesario, a la vez que recurso.

- Registro del proceso creativo.

- Producción artística. En este punto es importante remarcar, como lo indican la Dra. Natalia Calderón y el Dr. Fernando Hernández (2019), que investigación artística y producción artística no son equivalentes. Es muy común escuchar que se enuncia la idea de que toda producción conlleva investigación, sin embargo, no necesariamente se refiere al grado de profundización que plantea la investigación artística, razón por la cual la producción artística puede estar dentro de la investigación artística pero no a la inversa.

- Producción de conocimiento.

- Socialización.

- Reflexividad. 
Entrelazando caminos. Mis experiencias de colaboración

Todo lo anterior tendrá en consideración que se trata de una investigación de carácter sensible, singular y sin verdades absolutas, la cual ofrece una obra abierta, que bien puede ser colectiva en respuesta a su propia naturaleza. Se puede pensar que lo enlistado aquí ya era parte de la investigación relacionada con las artes — de hecho, el Dr. Iván Mejía apuntó en alguna ocasión: "la investigación artística se ha realizado siempre" (Mejía, 2014) - . Puede que haya algo de razón en ello, aunque como ya se mencionó anteriormente no hay que confundir investigación artística con producción artística. Si bien la segunda puede ser parte de la primera, la segunda no necesariamente tiene la intencionalidad de realizar este tipo de investigación. Lo que es un hecho es que las necesidades actuales determinadas por nuestro contexto parecen necesitar con cierta premura opciones de ejercicios investigativos que rompan con los esquemas rígidos en los que se ha estado trabajando, por lo cual la investigación artística no solo es pertinente, sino necesaria.

Imagen 2. Proceso creativo

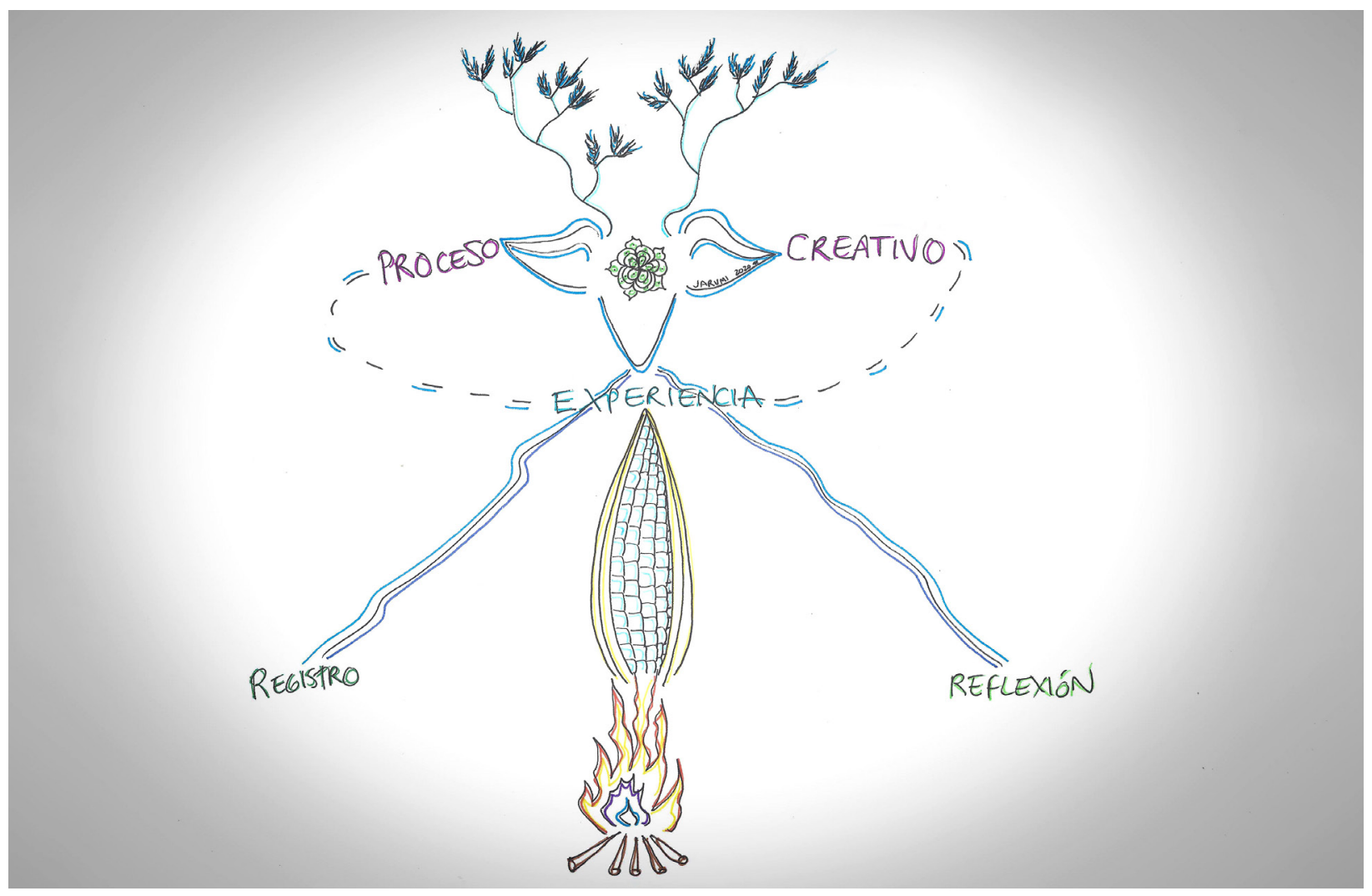

Fuente: Elaboración propia.

ESCENA. Revista de las artes, 2021, Vol. 81, Núm. 1 (julio-diciembre), pp. 304-319 
Volviendo a la lista previa, es oportuno desarrollar en específico el punto sobre el proceso creativo, dado que es un tema clave en el título de la asignatura que imparto. No está por demás puntualizar que sobre dicho tema versó parte de mi investigación de la maestría, en consecuencia, me permite compartir lo aprendido directo de la experiencia tanto en el ámbito de la producción artística como en el de investigadora y docente, el cual se ha mantenido como uno de mis principales intereses. A manera de registro de bitácora, se presenta la reflexión visual en la imagen 2, en la que se muestra la relación de elementos. En este caso con símbolos referentes a mi proyecto de investigación desarrollado en la maestría:

En la investigación artística, el proceso creativo se considera como un eje valioso al que le corresponde ser registrado. Tener conciencia de nuestro hacer y nuestro sentir y llevar un registro de ello es importante, porque - recordemos - se trata de un proceso conjunto. Una de las herramientas ideales para ello es la bitácora de trabajo, misma que yo utilicé durante el desarrollo de mi proyecto de investigación durante la maestría, así que, a partir de los enormes y favorables resultados que obtuve de dicho ejercicio, es que la solicito como parte de la evaluación en la clase del posgrado. Por esa razón, dedicamos tiempo a conversar algunos puntos que son esenciales para concretar su realización.

\section{¿Qué es la bitácora de trabajo?}

Es un acompañante inseparable, una herramienta de registro (o inclusive en ocasiones llega a ser la investigación en sí misma, eso depende de la intencionalidad del investigador).

\section{¿Para qué sirve la bitácora de trabajo?}

Sive para llevar nota de nuestro recorrido, de lo vivido, de lo investigado, de lo que nos mueve y nos significa, de lo que nos cuestiona. Puede incluir desde las notas bibliográficas hasta sueños, conversaciones, chistes, todo aquello que nos dice: ponme en tus notas. Nunca se sabe; es muy interesante como a la distancia, al recapitular, los detonantes y los hallazgos se hacen presentes y durante nuestros procesos investigativos y reflexivos llegan a ser determinantes.

\section{¿Qué caracteriza a la bitácora de trabajo?}

Se caracteriza por la libertad. Ahí se puede poner todo, el límite lo pone cada cual; lo esencial es darse la oportunidad de abrirse ante uno mismo. De inicio está pensada para que acompañe a quien investiga, así que no necesariamente será leída por otros, el autor tiene elección plena sobre a quién la muestra y qué de ella comparte. En las sesiones de re- 
visión, se indicó que pusieran un clip en las páginas que se consideraban privadas y, como acuerdo de respecto, los demás no leíamos esos apartados. Es importante mantener el ambiente de confianza, puesto que la bitácora puede llegar a ser un contenedor de lo más visceral de nuestras reflexiones y sentires.

Ahora bien, en el aspecto de que como parte de la clase es un elemento a evaluar, no se califica el contenido en sí, sino el hecho de realizar la actividad, de ver la constancia y la disciplina que ello implica; no es la cantidad, sino la calidad que involucra tiempo, trabajo y dedicación a tomar notas, desarrollar ideas. Además, en este caso, al ser el área de artes visuales, tener en cuenta la imagen es esencial para el desarrollo de las reflexiones, por lo que se solicitó tener un 50\% de reflexión visual, ya fuesen dibujos, bosquejos, fotografías, elementos gráficos u otros.

\section{¿Con qué fin se realiza la bitácora de trabajo?}

Se realiza con el propósito de llevar un registro de nuestros procesos, de tener evidencia material, para que al momento de realizar la recapitulación podamos ver y palpar el material. A veces no queda todo registrado al cien por ciento, pero el detalle que se haya plasmado servirá de referencia o detonante. Este en ocasiones genera una especie de ejercicio nemotécnico, que siempre nutrirá nuestros ejercicios de reflexión e indagación investigativa. Otro aspecto relevante es que se puede llevar más de una bitácora. Hay a quienes les funciona mejor llevar varias y las catalogan de acuerdo con sus intereses y necesidades; algunos optan por llevar una para las referencias bibliográficas, otra para los procesos de producción, otra para las ideas generales que surgen durante sus lecturas.

Asimismo, las características físicas de estas suelen ser también un factor importante, ya que los formatos o el tipo de papel permiten un manejo más adecuado según sea la necesidad, por ejemplo, una bitácora de tamaño menor al carta permite llevarla sin problema en el bolso y la inmediatez de escribir en cualquier lugar sería un factor a considerar; si se requiere bocetar con aguadas, un papel grueso será más idóneo. Estos son detalles que cada cual puede elegir y determinar qué le funciona más. Incluso, en ocasiones, puede ser aliciente de nuevas posibilidades, al resolver un planeamiento con lo que se tenga a la mano. A veces la libreta se eligió porque era atractiva a la vista, pero ya que se trabajó en ella sus propias características abrieron la posibilidad de ideas no previstas, por ejemplo, cuando el tipo de papel aporta cualidades de textura o color y eso detona reflexiones nuevas en quien lo usa. 
Algunos estudiantes han manifestado que ya habían trabajado bitácora con anterioridad y comentaron los grandes beneficios obtenidos; mientras que para quienes ha sido la primera vez, ha resultado una experiencia a veces complicada, debido a que se les olvida que la tienen y dejan pasar ideas, observaciones o sentires que luego no logran aterrizar del todo. Entonces, es cuando les digo: para eso es la bitácora, para que tengas como regresar sobre tus pasos y leerte, para que des tiempo y distancia a las reflexiones o sentires y logres verles con mayor claridad, identificar constantes, vislumbrar proyectos, definir conceptos o inclusive dejar de lado informaciones que no son pertinentes en el momento que se encuentre la investigación. Además, les permite realizar una revisión más personal sobre lo que les resulta significativo durante el proceso, ya sea en cuanto a los datos recopilados, los procesos artísticos-prácticos, los textos generados y las experiencias vividas.

Son varios los ejercicios realizados en las sesiones, todos pensados para compartir y generar retroalimentación, pero una de las estrategias más constantes, es el lograr inferir los conceptos más representativos de las lecturas asignadas para la clase. Hay que identificar cuáles son esos conceptos, dependiendo la intención de la sesión, los más importantes por el tema tratado y por la relación con el proyecto personal, una vez ubicados, hay que escribirlos en tarjetas para pegarlos en los muros y dialogarlos entre todos, ubicar las constantes y los contrastes, posteriormente, intentar armar un mapa en conjunto. He aquí una oportunidad para aplicar la idea de comunidad como la búsqueda de un fin derivado de intereses y prácticas en común, pues ya no se trata de identificar para uno mismo, sino pensando también en los demás, esto con el fin de lograr interacción e intercambio de ideas y reflexiones.

Si bien no se logró el cometido al primer intento de este ejercicio, ya que estaban acostumbrados a solo pensar en sí mismos, al día de hoy ${ }^{18}$, ya se tienen en cuenta unos a otros y se comparten información, intentan conjuntar reflexiones. Inclusive, ahora, cuando están en sus procesos individuales de investigación y topan con algo que consideran le sirve a otro, lo comparten. De igual manera, en las actividades en conjunto intentan dar espacio para la integración de las ideas del conjunto y no se cierran en sí mismos, es decir, se realiza una invitación hacer comunidad durante el proceso.

\footnotetext{
${ }^{18}$ He tenido la oportunidad de impartir clase por cuatro semestres consecutivos, es decir, de primero a cuarto semestre. Si bien se han integrado nuevos estudiantes, así como otros han dejado el grupo, los que se han mantenido han dado evidencia de dicho proceso derivado del compartir en comunidad.
}

ESCENA. Revista de las artes, 2021, Vol. 81, Núm. 1 (julio-diciembre), pp. 304-319 
La posibilidad de mirarse en el otro y escucharse a través de la voz de los demás es una herramienta para magnificar el diálogo. Es curioso como se puede tratar un mismo tema desde diferentes voces. Pensemos en la frase cada cabeza es un mundo y cada mundo se constituye de diversidad de elementos tanto divergentes como convergentes; se tienen elementos en común. Es por ello que pueden establecerse redes de intercambio y comunicación, sin embargo, no es solo una búsqueda de envío de información, de lo que se trata es de lograr conexión, una base humana cuyo entendimiento o posible comprensión derive de la empatía y la propia experiencia. Si bien, nos movemos en terrenos que la mayoría de las veces solicitan referencias teóricas, tenemos que traer a cuenta la relevancia de la percepción, la parte sensible que le da su cualidad a la experiencia estética y sobre la cual la producción artística tiene claramente una base.

Lo anterior es muy importante, ya que una de las mayores características de la investigación artística es la reflexividad. Esta solo es posible después de socializar la propuesta artística investigativa y de estar abierto al diálogo en conjunto para intercambiar puntos de vista al respecto para, posteriormente, hacer un ejercicio de revisión del propio trabajo y su proceso creativo, es decir, reflexionar sobre lo que resultó de lo reflexionado previamente, pero ya habiendo pasado por escrutinio de otro $u$ otros y de uno mismo. La posibilidad de abordar el tema del cómo impartir clase, siempre me ha parecido una oportunidad de retroalimentación, ya que uno comparte desde su experiencia las estrategias de socialización de la información, de intercambio de ideas y saberes. Asimismo, comparte las posibles estrategias pedagógicas derivadas de lo anterior, es decir, el cómo lograr enseñar y compartir a los estudiantes sobre aquello que nos es a fin, en un ambiente de comunidad.

\section{Recapitulando}

Compartir saberes es un compromiso a largo plazo

(Smith, 2016, p. 39).

La investigación artística es un proceso de aprendizaje que no requiere de una justificación, sino de llevar a cabo un ejercicio de reflexividad, en el cual es pertinente cuestionar las estructuras tanto de las decisiones como de los pensamientos que las generaron, las desarrollaron y las "materializaron". Es decir, aquellas que se pusieron en práctica: se accionó y no se quedó solo en la idea. Así, su principal intencionalidad es investigar para crear, donde la experiencia es crucial, ya que se realiza desde un proceso creativo reflexivo, que produ- 
cirá tanto propuesta artística como texto escrito; por ello es que podemos pensar en una actividad artística de acción. Hablamos de una investigación que da cuenta de un posible proceso cíclico generador de conocimiento, dado que producir puede devenir en teoría y la teoría devenir en producción. Lo anterior refiere a una doble implicación, donde el proceso creativo muestra un pensamiento crítico, el cual ejerce la voluntad y donde está presente una repercusión efecto-afecto que determina las formas que lo motivan. En otras palabras, manifiesta una base sentipensante que lleva a propuestas artísticas cuyo fundamento puede ser la interdisciplinariedad y que, a su vez, suelen ir dirigidas hacia el bien común, ya que son transversales y colaborativas, con lo que fomentan el hacer comunidad.

Se entiende, entonces, que la investigación artística sea considerada como campo de acción para la generación de conocimiento crítico, derivado principalmente del ejercicio de reflexividad que resulta de la relación con el entorno, la revisión del hacer, la socialización y la observación y el análisis no solo de los procesos, sino que también de los resultados y sus implicaciones. Para llevar a cabo estas acciones, la bitácora es un recurso ideal, ya que en ella se potencializa el registro (un aspecto esencial), la reflexión y por ende la investigación en sí misma.

Mi experiencia con la bitácora durante mi proceso creativo me permitió consolidar mi proceso de investigación en la maestría. También me dio la oportunidad de tomar conciencia de las reflexiones derivadas de mi convivencia en la comunidad Wixárika, lo cual da cuenta del ejercicio de reflexividad que caracteriza a la investigación artística. A la vez, me dio las bases necesarias para generar estrategias mediante las cuales poder compartir mis experiencias tanto personales como profesionales. Asimismo, en el plano docente, me ha permitido compartir con los estudiantes del posgrado lo aprendido e invitarles a experienciar de forma directa en sus propios procesos creativos. Aunado a ello, en conjunto hemos generado un ambiente propicio para el desarrollo de comunidad, en el cual se ha fomentado el intercambio de ideas, sentires y reflexiones que dan forma a sus proyectos y prácticas artísticas, lo cual tendrá como resultado sus respectivas tesis.

De igual manera, las anotaciones y registro de ideas y reflexiones derivadas de los diversos encuentros y actividades realizadas desde el Mitote ICDAC, en colaboración con otras instancias, han nutrido de sobremanera los alcances realizados hasta este momento en el proceso de indagación sobre este tema. El entrelazar los caminos de diversos grupos y sus integrantes ha desbordado en experiencias muy significativas que han nutrido mi proceso creativo. 
Entrelazando caminos. Mis experiencias de colaboración

Derivado de lo ya comentado y analizado hasta el momento, se puede inferir que la investigación artística es pertinente y necesaria en nuestro contexto actual. Esto debido a que las dinámicas de intercambio y de socialización de la información, así como las estrategias de los procesos de producción artística evidencian una demanda de metodologías propias del ámbito de la investigación en el área de las artes. A manera de registro de bitácora, se presenta la reflexión visual en la imagen 3 en la que se muestra el territorio recorrido por los diversos conceptos abordados en el artículo.

Imagen 3. Cartografía del territorio

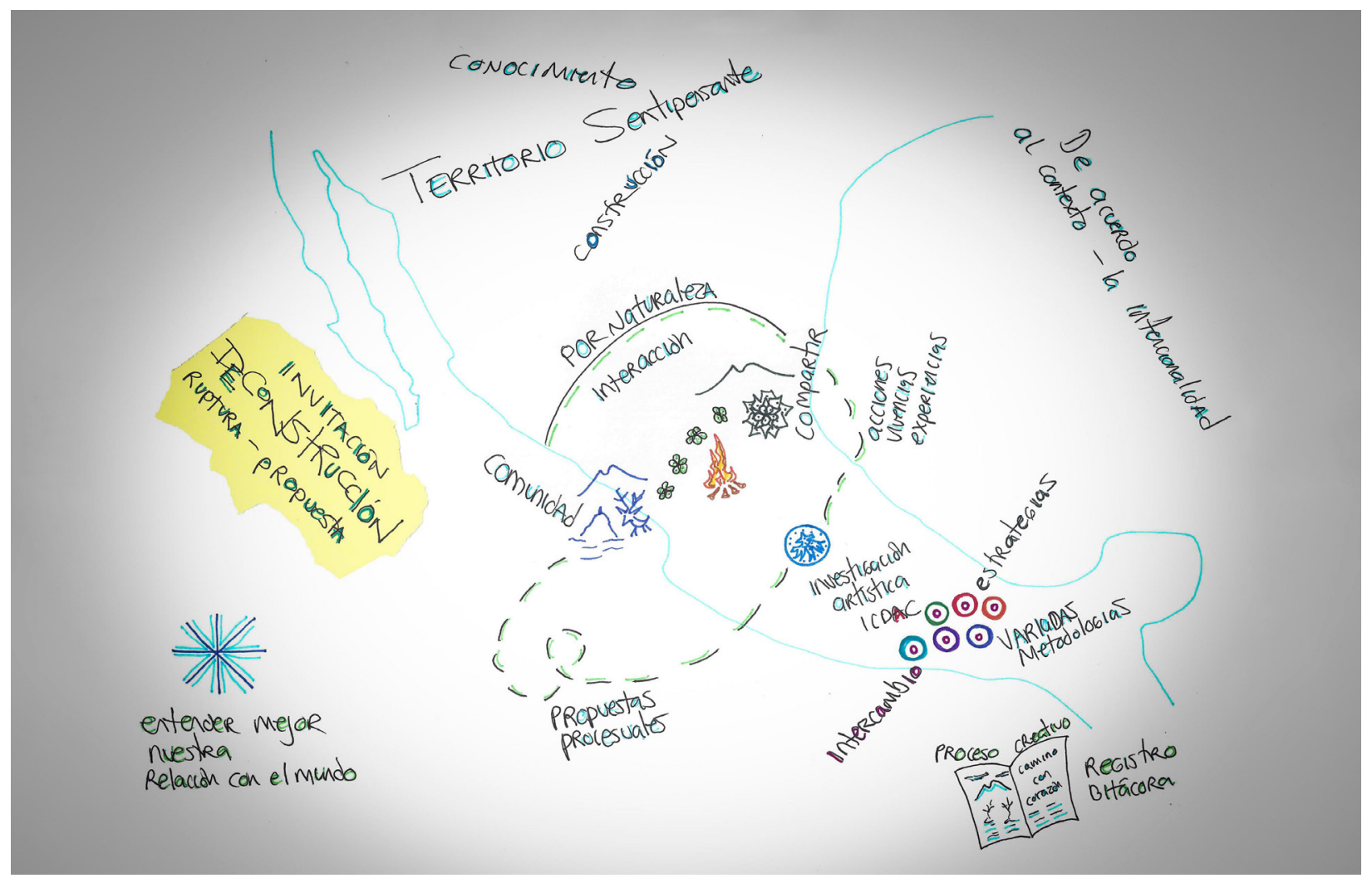

Fuente: Elaboración propia. 


\section{Referencias}

Battell Lowman, E. \& Barker, A. (s.f.). Global Social Theory. Smith, Linda Tuhiwai. [Página web]. Recuperado de https://globalsocialtheory.org/thinkers/linda-tuhiwai-smith/

Borgdorff, H. (2019). El debate sobre la investigación en las artes. Recuperado de https:// issuu.com/fwalita/docs/henk_borgdorff._el_debate_sobre_la_

Diccionario de la Real Academia Española. (2021). Deconstruir. Recuperado de: https://dle. rae.es/deconstruir?m=form

Calderón, N. \& Hernández, F. (2019). La investigación artística. Un espacio de conocimiento disruptivo en las artes y en la universidad. Barcelona: Octaedro.

Dávila López, A. (2015). Sobre cómo la mirada mágica pone en escena al ser, a la vez que éste se devela en el proceso creativo (Tesis de maestría). Universidad Nacional Autónoma de México, México. Recuperado de https://repositorio.unam.mx/contenidos/3525822

Mejía, I. (2014). El artista como investigador. Recuperado de https://lasdisidentes. com/2015/03/30/el-artista-como-investigador-la-docencia-como-proceso-emancipatorio-y-la-escritura-como-ejercicio-politico/

Ruíz, B. C. \& Torres, L. C. (2016). Pensamiento complejo y creatividad. Revista Ontare, 4(2), 7-26. doi: 10.21158/23823399.v4.n2.2016.1625

Smith, L.T. (2016). A descolonizar metodologías. Investigación y pueblos indígenas. Santiago de Chile: LOM.

Vera, S. (2004). Proyecto artístico y territorio. Granada: Universidad de Granada.

Vivas, G. (09 de mayo de 2020). Codesarrollo sentipensante para un desarrollo sostenible. Levante. El mercantil valenciano. Recuperado de https://www.levante-emv.com/opinion/2020/05/09/codesarrollo-sentipensante-desarrollo-sostenible-11485572.html

Xirau, R. (2003). Introducción a la historia de la filosofía. México: Universidad Nacional Autónoma de México. 